\title{
Measurement of high time-bandwidth pulses on a chip with SPIDER
}

\author{
Alessia Pasquazi, ${ }^{* 1}$ Marco Peccianti, ${ }^{1,2}$ Roberto Morandotti ${ }^{1}$, Jose Azana ${ }^{1}$, and David J. Moss ${ }^{3}$ \\ ${ }^{1}$ INRS Énergie, Matériaux et Télécommunications, 1650 Blvd. Lionel Boulet, Varennes (Québec), J3X 1S2 Canada; \\ ${ }^{2}$ Institute for Complex Systems - CNR, UOS Montelibretti, Via dei Taurini 19, 00185 Roma, Italy; \\ ${ }^{3}$ CUDOS, Institute of Photonics and Optical Science (IPOS), School of Physics, University of Sydney, Sydney,
} Australia.

Received February 21, 2012; accepted March 28, 2012; published March 31, 2012

\begin{abstract}
We develop a method capable of characterizing both the amplitude and phase of ultrafast optical pulses with the aid of a synchronized incoherently-related clock pulse based on a novel variation of SPIDER. It exploits degenerate four-wave-mixing and its design is amenable for full "on chip" signal characterization. By implementing our method in a CMOS compatible waveguide, we measure pulses with $<100 \mathrm{~mW}$ peak power, a frequency bandwidth > $1 \mathrm{THz}$, and up to $100 \mathrm{ps}$ pulsewidths. Our novel reconstruction algorithm that we call Fresnel-Limited Extraction Procedure (FLEA), yields a time-bandwidth product (TBP) $>100$.
\end{abstract}

The accurate measurement of the amplitude and the phase of an ultrafast optical signal [1-2] is of primary importance for many fields such as metrology and coherent optical telecommunications. In the latter case the high repetition rates are now approaching the inherent bandwidth limits of electronic circuits [2], and the need of an effective and ultrafast method to characterize the optical data stream is compelling, requiring the development of new and effective approaches to characterize the optical signal by means of all optical methods[1-2], preferably in integrated platforms [4-6].

The complex character (amplitude and phase) of the electric field in pulses is very often measured in optics by means of interferometric techniques [2]. Spectral sheared interferometry is a popular method, widely used in both electrical and all optical implementations, which employs as reference a replica of the pulse itself shifted (sheared) in frequency [2]. The resulting interferogram of this selfreferenced approach enables the unambiguous reconstruction of the amplitude and phase of the pulse.

Spectral Phase Interferometry for Direct Electric-Field Reconstruction or SPIDER is the most popular implementation of spectral shared interferometry, introduced by Iaconis and Wamsley in 1998 [7]. Its success is due to its intrinsically ultrafast [8] and singleshot [9] nature as well as to the simple, direct and robust procedure it uses for phase retrieval [2, 7]. SPIDER is

\footnotetext{
*E-mail: alessia.pasquazi@gmail.com
}

usually based on three wave mixing (TWM) interaction of a strongly chirped pump with two delayed replicas of the pulse under test (PUT). Typically the pump is derived from the PUT [7], making the technique intrinsically selfreferencing. When an independent, well characterized pump pulse is used the method improves in terms of accuracy and reliability [10], and this method is commonly referred to as X-SPIDER $[2,11]$.

Here, we discuss a novel approach for the characterization of both amplitude and phase of ultrafast optical pulses that can work directly in a fiber network. It is based on a novel variation of X-SPIDER using four-wave-mixing (FWM) in a nonlinear waveguide.

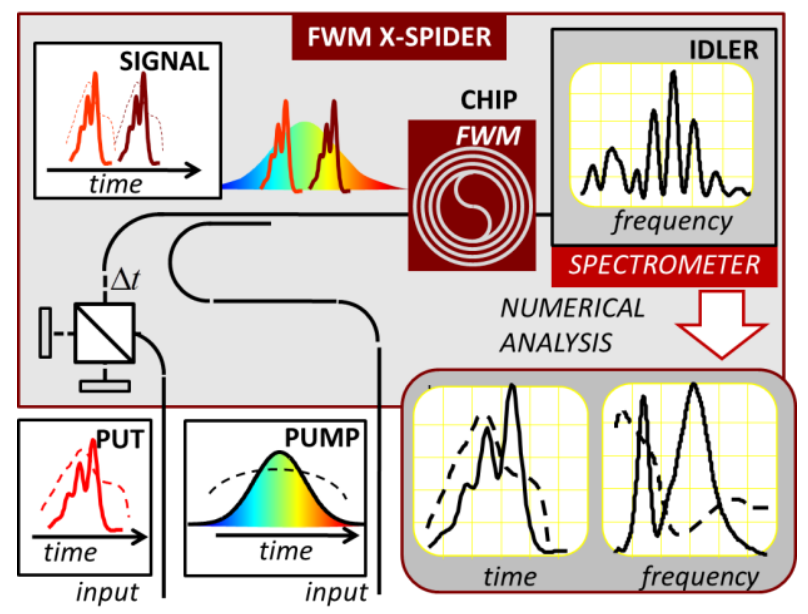

Fig 1. Experimental setup of the X-SPIDER based on FWM in a CMOS compatible waveguide.

Our method (Fig. 1) maintains the role of the signal, of the (external) pump and of the idler, associated with the classical TWM implementation. Unlike conventional $\chi^{(2)}$ based approaches to optical phase measurements, our $\chi^{(3)}$ based method is applicable to centro-symmetric materials such as glass and silicon, important platforms for photonics integration. Moreover, we introduce a novel 

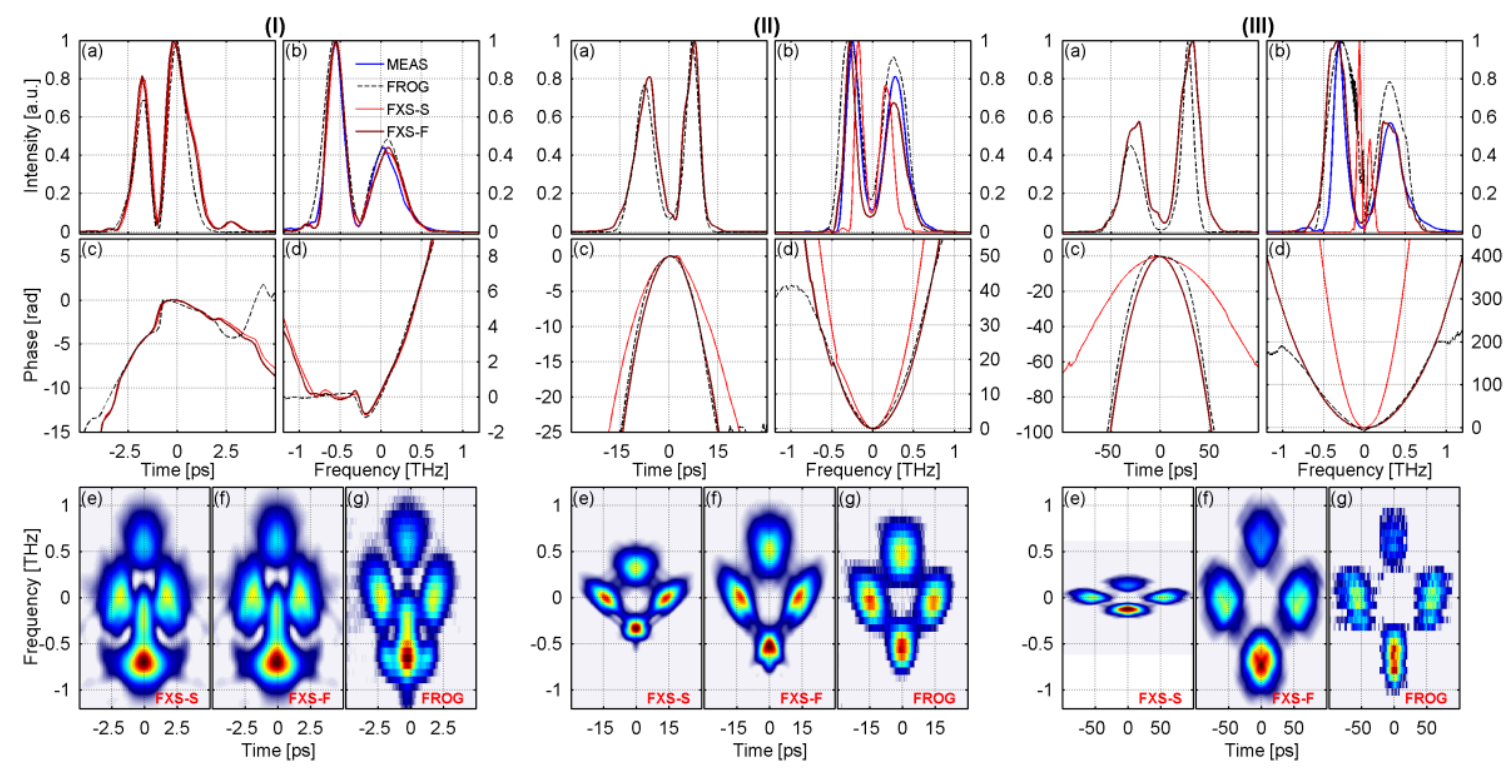

Fig. 2. Measurement of optical pulses performed with the FWM-X-SPIDER device, for a complex waveform of $\sim 1$ THz bandwidth: retrieved waveform from spectra in Fig. (2) and comparison with experimentally measured FROG SHG spectrograms (after Ref. [6]). Panel (I), (II) and (III) display pulses with a time-bandwidth product 5, 30 and 100 respectively. For each panel, the amplitude (a, b) and the phase (c, d) are reconstructed in the temporal and spectral domains, respectively. The red and brown lines depict the results obtained through the application of the standard (FXS-S) and novel (FLEA) (FXS-F) phase-reconstruction algorithms on the measured spectral interferogram, respectively, while the dashed curves are the profiles extracted from the SHG-FROG measurement. In (b) the directly measured spectrum is also shown (in blue). For each panel, (e)-(f) show the numerically reconstructed FROG spectrograms for the pulse profiles retrieved from the standard and the novel phase-reconstruction algorithms, respectively, while (g) reports the SHG-FROG measurements performed to characterize the same PUT.

extraction algorithm that significantly extends the time bandwidth product (TBP) of the measurable pulses that works with any implementation of an X-SPIDER method without requiring any modification of the experimental setup.

SPIDER implementations that exploit nonlinear optical methods typically rely on the approximation that the chirp of the pump is much wider than that of the PUT. When this condition is violated, the information cannot be retrieved with the usual algorithm. For this reason, SPIDER has been traditionally limited to pulses with low TBP. It is interesting to note though that the nonlinear product still contains information on the phase of the PUT, and so some strategies have been developed to overcome this limitation, restricted to Gaussian pulses in the self-referenced case [12]. To overcome the above constraints, we introduce a novel and general approach for the phase extraction, which we term a Fresnel-limited extraction algorithm (FLEA). This approach completely removes any restriction on the PUT waveform and can be generally applied to any implementation of SPIDER methods that employ an external pump reference (i.e. XSPIDER). Most importantly, it also works for highly chirped pulses, significantly extending the operating range of an X-SPIDER device.

After Ref. [6], we experimentally discuss our approach using a 45-cm long spiral high index as the nonlinear optical element, consisting of a doped silica glass waveguide in a CMOS compatible platform [13-14]. This implementation of our X-SPIDER based on FWM is sensitive to pulse energies as low as $5 \mathrm{pJ}$.

The nonlinear interaction considered here is degenerate FWM - a process involving three waves: a pump $p(t)$ that amplifies a signal $s(t)$ and generates an idler $g(t)$. The pump pulse is shaped in order to possess a quadratic temporal phase curvature and an approximately constant envelope during the nonlinear interaction. Such a shape can be easily obtained by temporally stretching a transform-limited pulse with a smoothly varying flat-phase spectrum, e.g. a bell-shaped, or spectrally flat-top, spectrum. The pump propagates through a predominantly first-order dispersive element defined by the total dispersion $\phi_{P}$. For the sake of simplicity, the pump pulse envelope is assumed to be constant over the entire duration of the two delayed PUT pulses, i.e. $p(t) \approx$ $\exp \left(i t^{2} / \phi_{P}\right)$. The signal $s(t)=[e(t+\Delta t / 2)+e(t-\Delta t / 2)]$ consists of two replicas of the PUT $e(t)$ delayed by $\Delta t$. The idler induced by the degenerate FWM interaction is simply given by the following expression [6]:

$$
\begin{aligned}
& \overline{g(t)} \propto[e(t+\Delta t / 2)+e(t-\Delta t / 2)] \overline{p(t)^{2}} \\
& \approx[e(t+\Delta t / 2)+e(t-\Delta t / 2)] \exp \left(-i t^{2} / \phi_{P}\right)
\end{aligned}
$$


where the upper bar stands for the complex conjugate. We define $f_{e}(t)$ as the Fresnel integral of the PUT corresponding to its dispersion through half the length of the dispersive element, i.e.

$$
\begin{aligned}
& f_{e}(t)=\exp \left(-i t^{2} / \phi_{P}\right) . \\
& \cdot \int_{-\infty}^{\infty} e(x) \exp \left(-i x^{2} / \phi_{P}+2 i t x / \phi_{P}\right) d x
\end{aligned}
$$

It is possible to verify that Eq. (1) can be expressed in the spectral domain as a function of the Fresnel integral:

$$
|G(\omega)|^{2} \propto\left|f_{e}\left(-\omega \phi_{P} / 2+\Delta t / 2\right)+f_{e}\left(-\omega \phi_{P} / 2-\Delta t / 2\right)\right|^{2}(3)
$$

In FLEA we use the exact interferogram pattern defined by relation (3) to extract the PUT spectral phase information. It is important to stress that under the usual approximation where the X-SPIDER is applied - i.e. for a PUT much shorter in time than the pump - the Fresnel integral (2) is proportional to the spectral shape of the PUT $E(\omega)$, i.e. $f_{e}(t) \approx E\left(2 t / \phi_{P}\right) \exp \left(-i t^{2} / \phi_{P}\right)$, and the classical expression for the spectral-sheared interference pattern, typically used for phase recovery in SPIDER, can be derived.

The experimental setup is shown in Fig. 1. Pump and pulse under test (PUT) pulses were prepared from a $17 \mathrm{MHz}$ mode-locked fibre source, providing pulses at $\lambda=1530 \mathrm{~nm}$. The chirped pump was prepared via the spectral broadening of the pulses generated from a modelocked fibre laser in a nonlinear fibre, followed by filtering with a detuned $10 \mathrm{~nm}$ super-gaussian-like bandpass optical filter centred at $1550 \mathrm{~nm}$, temporal stretching through a $1 \mathrm{~km}$ length of linearly dispersive SMF and finally by amplification with an EDFA up to 1nJ. A 3-dB interferometer split the PUT into two identical copies, and a movable mirror controlled their relative delay. The two replicas of the PUT (i.e the signal) and the pump were coupled into the 45-nm waveguide. We prepared a PUT through self-phase-modulation induced spectral broadening, with a bandwidth of $\sim 1 \mathrm{THz}$. The pulse energy at the input of the chip was $<5 \mathrm{pJ}$ and we applied different spools of standard single-mode fire (SMF) to control the chirp, stretching the pulse up to $100 \mathrm{ps}$, corresponding to $\sim 70 \mathrm{~mW}$ peak power. Fig. 2 shows the full set of results for our FWM X-SPIDER measurements using both the classic algorithm and FLEA, along with measurements using a SHG-based FROG technique. As expected, for low TBP pulses (i.e., the short-pulse regime) our FWM X-SPIDER approach yielded the same solution when using either phase recovery algorithms (Fig. 2, Panel (I), (e, f)) and both of these agreed well with the experimental FROG spectrogram (Fig. 2, Panel (I), (g)). For large TBP (highly chirped, long pulsewidth) pulses the standard phaserecovery algorithm yielded a pulse spectrogram profile that deviated significantly from the experimental FROG trace - the frequency scale being compressed compared to the FROG trace. On the other hand, the spectrogram obtained using the FLEA agreed very well with the FROG trace, thus confirming the extended range of TBP operation for our FWM X-SPIDER device.

We report the demonstration of an integrated optical oscilloscope capable of measuring both the phase and amplitude of optical pulses over time windows as long as 100 ps and with a bandwidth up to $\sim 1 \mathrm{THz}$. This device is based on a novel adaptation of the X-SPIDER technique, relying on FWM rather than on conventional TWM, and using an integrated CMOS compatible chip. We successfully characterized both sub-picosecond and highly stretched optical pulses in amplitude and phase, at pulse energies $<5 \mathrm{pJ}$ This was achieved with the aid of our extended numerical phase-recovery method (FLEA), which allowed us to exceed significantly the typical operating regime of standard X-SPIDER in terms of both pulse duration and chirp.

\section{References}

[1] R. Trebino, Frequency Resolved Optical Gating: the Measurement of Ultrashort Optical Pulses (Kluwer Academic, 2002).

[2] I.A. Walmsley and C. Dorrer, Adv. Opt. Photon. 1, 308 (2009); http://www.opticsinfobase.org/abstract.cfm?URI=aop-1-2-308.

[3] Nature Photonics Workshop on the future of optical communication,Tokyo, Oct. 2007

[4] M.A. Foster et al., Nature 456, 81(2008); http://www.nature.com/nature/journal/v456/n7218/full/nature07430.ht $\mathrm{ml}$.

[5] E. K. Tien, X. Z. Sang, F. Qing, Q. Song, and O. Boyraz., Appl. Phys. Lett. 95, 051101 (2009); http://apl.aip.org/resource/1/applab/v95/i5/p051101_s1.

[6] A. Pasquazi et al., Nature Photon. 5, 618 (2011); http://www.nature.com/nphoton/journal/v5/n10/full/nphoton.2011.199. html.

[7] C. Iaconis and I.A. Walmsley, Opt. Lett. 23, 792 (1998); http://www.opticsinfobase.org/abstract.cfm?URI=ol-23-10-792.

[8] L. Gallmann et al., Opt. Lett. 24, 1314 (1999); http://www.opticsinfobase.org/ol/abstract.cfm?URI=ol-24-181314.

[9] C. Dorrer et al., Opt. Lett. 24, 1644 (1999); http://www.opticsinfobase.org/ol/abstract.cfm?URI=ol-24-221644.

[10] D. Keusters et al., J. Opt. Soc. Am. B 20, 2226 (2003); http://www.opticsinfobase.org/josab/abstract.cfm?URI=josab-2010-2226.

[11] M. Hirasawa et al., Appl. Phys. B 74, S225 (2002); http://www.springerlink.com/content/ub632vh08c712bfv.

[12] J. Wemans, G. Figueira, N. Lopes and L. Cardoso, Opt. Lett. 31, 2217 (2006); http://www.opticsinfobase.org/ol/abstract.cfm?URI=ol-31-142217.

[13] M. Peccianti et al., Opt. Expr. 18, 7625 (2010); http://www.opticsinfobase.org/oe/abstract.cfm?URI=oe-18-87625.

[14] A. Pasquazi et al., Opt. Expr. 18, 3858 (2010); http://www.opticsinfobase.org/oe/abstract.cfm?URI=oe-18-43858 . 